\title{
Jet substructure and correlations in hadronic final states from ALICE
}

\section{James Mulligan for the ALICE Collaboration ${ }^{a, b, *}$}

${ }^{a}$ Nuclear Science Division, Lawrence Berkeley National Laboratory, Berkeley, California 94720, USA

${ }^{b}$ Physics Department, University of California, Berkeley, CA 94720, USA

E-mail: james.mulligan@berkeley.edu

Jets of high energy collimated particles provide a rich phenomenology to study quantum chromodynamics, from first-principles tests of perturbative calculations to investigations of the emergent properties of the strongly-coupled quark-gluon plasma. In these proceedings, we highlight several recent jet measurements by the ALICE Collaboration, with a focus on jet substructure observables.

The Ninth Annual Conference on Large Hadron Collider Physics - LHCP2021

7-12 June 2021

Online

${ }^{*}$ Speaker 


\section{Introduction}

Jet observables in both proton-proton and heavy-ion collisions can be used to study fundamental aspects of quantum chromodynamics (QCD). In pp collisions, jet measurements test state-of-the-art perturbative calculations and explore the transition from the perturbative to the nonperturbative regimes. In $\mathrm{Pb}-\mathrm{Pb}$ collisions, jets serve as probes of the quark-gluon plasma (QGP) to study the physical properties of deconfined QCD matter [1-4]. In both cases, studying the internal pattern of particles within jets, known as jet substructure, enables the design of observables to target specific regions of QCD phase space [5].

In these proceedings, we highlight a selection of recent results from the ALICE experiment [6], with an emphasis on analytically calculable jet substructure observables. All results presented utilize jets reconstructed from charged particles at midrapidity using the anti- $k_{\mathrm{T}}$ algorithm [7], and are corrected for detector effects and (in $\mathrm{Pb}-\mathrm{Pb}$ collisions) underlying event fluctuations.

\section{Jet measurements in proton-proton collisions}

Jet angularities. The infrared and collinear safe jet angularities [8,9] provide a flexible way to study QCD in both $\mathrm{pp}$ and $\mathrm{Pb}-\mathrm{Pb}$ [10-12] collisions by systematically varying the weight of collinear radiation via the parameter $\alpha>0$ :

$$
\lambda_{\alpha}=\sum_{i \in \mathrm{jet}}\left(\frac{p_{\mathrm{T}, i}}{p_{\mathrm{T}, \mathrm{jet}}}\right)\left(\frac{\Delta R_{i}}{R}\right)^{\alpha} .
$$

ALICE recently measured the ungroomed, and, for the first time, the groomed jet angularities in pp collisions for a variety of $\alpha$ [13], shown in Fig. 1. The distributions are compared to SCET calculations [14, 15] using a Monte Carlo (MC) based folding procedure, and reveal a transition from generally good agreement in the perturbative regime to deviation in the nonperturbative regime.

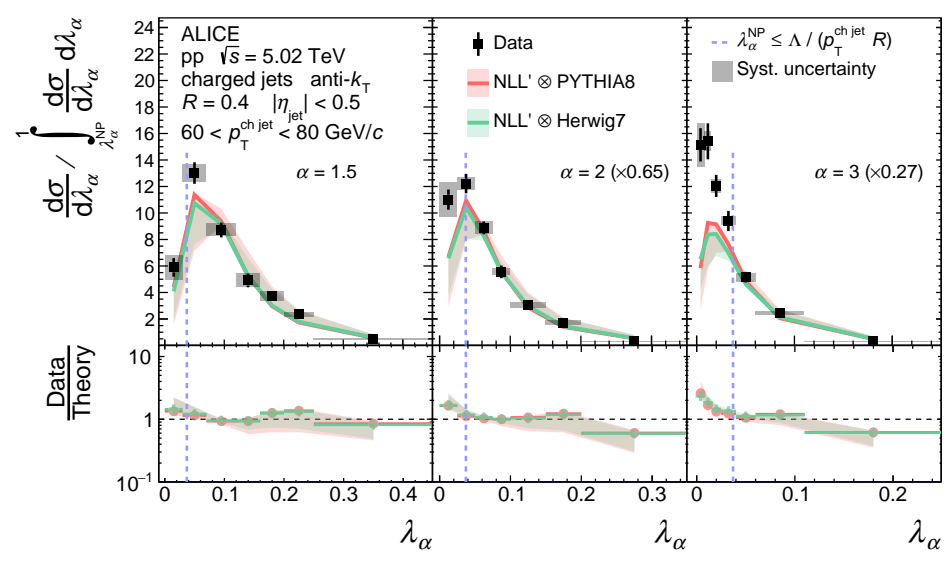

Figure 1: Comparison of measured ungroomed jet angularities $\lambda_{\alpha}$ in pp collisions for $\alpha=1.5,2,3$ to analytical NLL' predictions [14] with MC hadronization and charged particle corrections [13]. 
Jet axis differences. The soft, wide-angle substructure of jets can be studied by comparing the jet-by-jet rapidity-azimuth difference between pairs of jet axes:

$$
\Delta R_{\mathrm{axis}}=\sqrt{\Delta y^{2}+\Delta \varphi^{2}}
$$

where the axes can be defined by (i) the standard $E$-scheme recombination axis, (ii) the Soft Drop (SD) groomed axis, or the (iii) winner-take-all (WTA) recombination axis [16]. Figure 2 (left) shows the first measurement of these pairwise axis differences, where the comparison of the standard and SD axes shows small absolute differences which increase as the grooming condition becomes larger. These soft-sensitive observables can be used to study a variety of nonperturbative physics [16].

\section{Jet measurements in heavy-ion collisions}

Subjet fragmentation. In heavy-ion collisions, measurements of reclustered subjets have been proposed as sensitive probes of jet quenching [19, 24, 25]. We consider first inclusively clustering jets with the anti- $k_{\mathrm{T}}$ jet algorithm and jet radius $R$, and then reclustering the jet constituents with the anti- $k_{\mathrm{T}}$ jet algorithm and subjet radius $r<R$. We then consider the fraction of transverse momentum carried by the subjet compared to the initial jet: $z_{\mathrm{r}}=p_{\mathrm{T}}^{\text {ch subjet }} / p_{\mathrm{T}}$, jet. Figure 2 (right) shows the distribution of leading subjets with $r=0.1, R=0.4$ in both pp and $\mathrm{Pb}-\mathrm{Pb}$ collisions. The distributions are compared to theoretical predictions [19-23] which accurately reproduce a mild rising trend of the ratio with $z_{\mathrm{r}}$, which can be attributed to jet collimation, which then falls as $z_{\mathrm{r}} \rightarrow 1$, which may be due to the large quark/gluon fraction at $z_{\mathrm{r}} \rightarrow 1$. These measurements offer an opportunity to probe higher $z$ than hadron fragmentation measurements, and are an important ingredient for future tests of the universality of in-medium jet fragmentation functions.
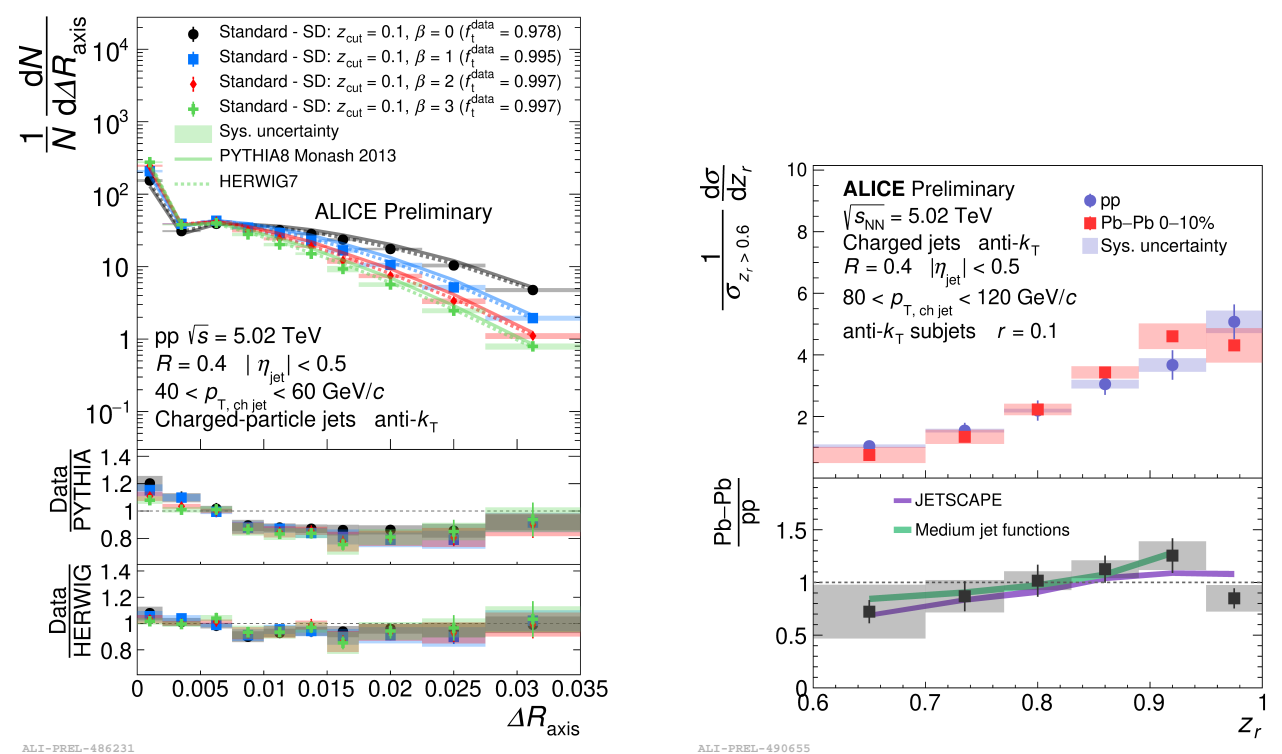

Figure 2: Left: ALICE measurements of pairwise jet axis angular differences for a variety of Soft Drop grooming conditions, compared to PYTHIA [17] and HERWIG [18]. Right: ALICE measurements of leading subjet fragmentation in $\mathrm{pp}$ and $\mathrm{Pb}-\mathrm{Pb}$ collisions, compared to theoretical predictions [19-23]. 

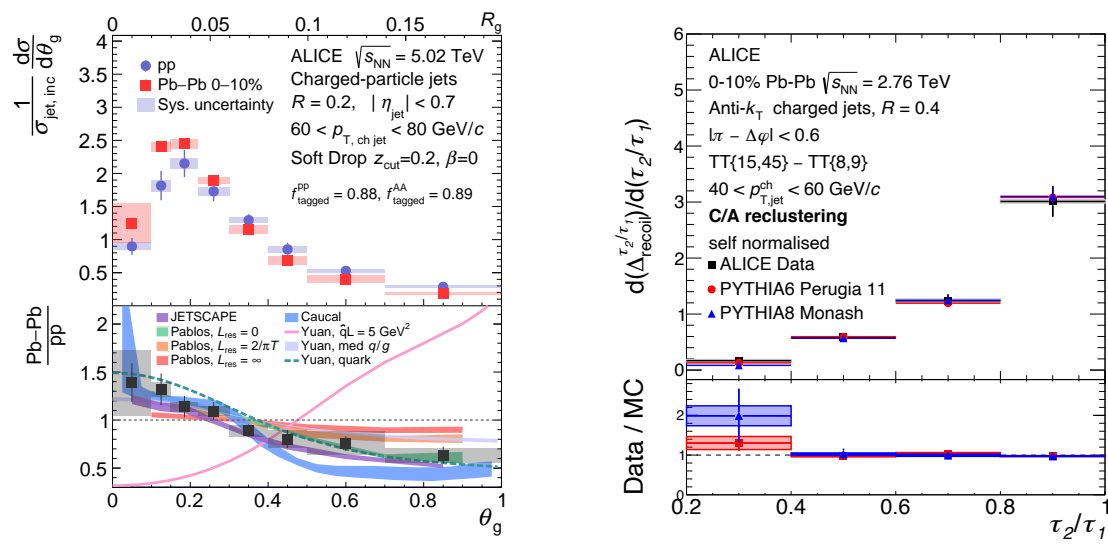

Figure 3: Left: Measurements of $\theta_{\mathrm{g}}$ in $\mathrm{Pb}-\mathrm{Pb}$ compared to pp collisions [43]. Right: Measurements of the $\tau_{2} / \tau_{1} \mathrm{~N}$-subjettiness distribution in $\mathrm{Pb}-\mathrm{Pb}$ collisions [47] compared to PYTHIA [17].

Groomed jet radius. Jet grooming techniques [26-28] have been applied to heavy-ion collisions to explore whether jet quenching modifies the hard substructure of jets [29-39]. By using strong grooming conditions [40], ALICE measured the groomed momentum fraction, $z_{\mathrm{g}}$ [41], and the groomed jet radius, $\theta_{\mathrm{g}}$ [42], with the Soft Drop grooming algorithm. Figure 3 (left) shows a narrowing of the $\theta_{\mathrm{g}}$ distributions in $\mathrm{Pb}-\mathrm{Pb}$ collisions relative to $\mathrm{pp}$ collisions [43]. These measurements are compared to a variety of jet quenching models [21-23, 29, 31, 33-35, 44-46], most of which capture the qualitative narrowing effect observed. This behavior is consistent with models implementing an incoherent interaction of the jet shower constituents with the medium, but is also consistent with medium-modified quark/gluon fractions with fully coherent energy loss presenting the opportunity for future measurements to disentangle them definitively.

$N$-subjettiness. Semi-inclusive hadron-jet correlations are well-suited to statistical background subtraction procedures in heavy-ion collisions, which allows jet measurements to low $p_{\mathrm{T}}$ and large $R[48,49]$. Recently, ALICE measured the N-subjettiness [50, 51] of jets recoiling from a high- $p_{\mathrm{T}}$ hadron with this method [47]. Figure 3 (right) shows the distribution of per-trigger semi-inclusive yields of the $\tau_{2} / \tau_{1}$ ratio in $\mathrm{Pb}-\mathrm{Pb}$ collisions compared to PYTHIA [17], which show no significant modification in the pronginess of jets in heavy-ion collisions. This suggests that medium-induced emissions are not sufficiently hard to produce a distinct secondary prong, in line with the lack of modification of $z_{\mathrm{g}}$ observed [43].

\section{Conclusion}

We have presented several new ALICE measurements of jet substructure in pp collisions, which provide new tests of our first-principles understanding of QCD by exploring the transition from perturbative to nonperturbative physics. In heavy-ion collisions, ALICE measurements are producing an emerging picture of jet quenching phenomenology: hard splittings are not strongly modified, as evidenced by $z_{\mathrm{g}}, \tau_{N}$, but there is a strong collimation or filtering effect of wide jets, as evidenced by $\theta_{\mathrm{g}}$. The medium-induced soft splitting responsible for this filtering may be exposed in regions dominated by quark jets, as suggested by high- $z_{\mathrm{r}}$ subjet fragmentation. Together, these observables offer future prospects to constrain physical properties of the QGP using global analyses. 


\section{References}

[1] G.-Y. Qin and X.-N. Wang, Int. J. Mod. Phys. E 24 (2015), 1530014.

[2] J.-P. Blaizot and Y. Mehtar-Tani, Int. J. Mod. Phys. E 24 (2015), 1530012.

[3] A. Majumder and M. van Leeuwen, Prog. Part. Nucl. Phys. 66 (2011), 41.

[4] JETSCAPE collaboration, S. Cao et al., Phys. Rev. C 104 (2021), 024905 [2102 . 11337].

[5] A. J. Larkoski, I. Moult and B. Nachman, Physics Reports 841 (2020), 1-63.

[6] ALICE Collaboration, J. Instrum. 3 (2008), S08002.

[7] M. Cacciari, G. P. Salam and G. Soyez, JHEP 04 (2008), 063.

[8] L. G. Almeida, S. J. Lee, G. Perez, G. F. Sterman, I. Sung and J. Virzi, Phys. Rev. D 79 (2009), 074017 [0807. 0234].

[9] A. J. Larkoski, J. Thaler and W. J. Waalewijn, JHEP 2014 (2014), 129.

[10] ATLAS Collaboration, PRD 86 (2012), 072006.

[11] CMS Collaboration, PRD 98 (2018), 092014.

[12] ALICE Collaboration, JHEP 2018 (2018), 139.

[13] ALICE collaboration, S. Acharya et al., 2107.11303.

[14] Z.-B. Kang, K. Lee and F. Ringer, JHEP 2018 (2018), 110.

[15] Z.-B. Kang, K. Lee, X. Liu and F. Ringer, PLB 793 (2019), 41.

[16] P. Cal, D. Neill, F. Ringer and W. J. Waalewijn, JHEP 04 (2020), 211 [1911. 06840].

[17] T. Sjostrand and et al., CPC. 191 (2015), 159.

[18] J. Bellm et al., Eur. Phys. J. C 76 (2016), 196 [1512.01178].

[19] Z.-B. Kang, F. Ringer and W. J. Waalewijn, JHEP 07 (2017), 064 [1705. 05375].

[20] J.-W. Qiu, F. Ringer, N. Sato and P. Zurita, PRL 122 (2019), 252301.

[21] J. H. Putschke et al., 1903.07706.

[22] Y. He, T. Luo, X.-N. Wang and Y. Zhu, PRC 91 (2015), 054908.

[23] A. Majumder, $P R C \mathbf{8 8}$ (2013), 014909.

[24] D. Neill, F. Ringer and N. Sato, JHEP 07 (2021), 041 [2103. 16573].

[25] L. Apolinário, J. G. Milhano, M. Ploskon and X. Zhang, Eur. Phys. J. C 78 (2018), 529 [1710.07607].

[26] A. J. Larkoski, S. Marzani, G. Soyez and J. Thaler, JHEP 05 (2014), 146.

[27] M. Dasgupta, A. Fregoso, S. Marzani and G. P. Salam, JHEP 09 (2013), 029.

[28] A. J. Larkoski, S. Marzani and J. Thaler, PRD. 91 (2015), 111501.

[29] Y.-T. Chien and I. Vitev, PRL 119 (2017), 112301.

[30] Y. Mehtar-Tani and K. Tywoniuk, JHEP 2017 (2017), 125.

[31] N.-B. Chang, S. Cao and G.-Y. Qin, PLB 781 (2018), 423-432.

[32] R. Kunnawalkam Elayavalli and K. C. Zapp, JHEP 7 (2017), 141.

[33] P. Caucal, E. Iancu and G. Soyez, JHEP 10 (2019), 273.

[34] F. Ringer, B.-W. Xiao and F. Yuan, PLB 808 (2020), 135634.

[35] J. Casalderrey-Solana, G. Milhano, D. Pablos and K. Rajagopal, JHEP 01 (2020), 044.

[36] H. A. Andrews and et al., JPG 47 (2020), 065102.

[37] CMS Collaboration, PRL 120 (2018), 142302.

[38] ALICE Collaboration, PLB 802 (2020), 135227.

[39] CMS Collaboration, JHEP 2018 (2018), 161.

[40] J. Mulligan and M. Ploskon, Phys. Rev. C 102 (2020), 044913 [2006. 01812].

[41] P. Cal, K. Lee, F. Ringer and W. J. Waalewijn, 2106.04589.

[42] Z.-B. Kang, K. Lee, X. Liu, D. Neill and F. Ringer, JHEP 2020 (2020), 54.

[43] ALICE collaboration, S. Acharya et al., 2107.12984.

[44] P. Caucal, E. Iancu, A. Mueller and G. Soyez, PRL 120 (2018), 232001.

[45] J. Casalderrey-Solana, D. C. Gulhan, J. G. Milhano, D. Pablos and K. Rajagopal, JHEP 019 (2014), 019.

[46] Z. Hulcher, D. Pablos and K. Rajagopal, JHEP 010 (2018), 10.

[47] ALICE collaboration, S. Acharya et al., 2105.04936.

[48] ALICE Collaboration, JHEP 2015 (2015), 170.

[49] STAR Collaboration, PRC 96 (2017), 024905.

[50] I. W. Stewart, F. J. Tackmann and W. J. Waalewijn, Phys. Rev. Lett. 105 (2010), 092002 [1004 . 2489].

[51] J. Thaler and K. Van Tilburg, JHEP 03 (2011), 015 [1011.2268]. 